\title{
ON NEUMANN'S FORMULA FOR THE LEGENDRE FUNCTIONS
}

\author{
by T. M. MACROBERT
}

(Received 29th November, 1948)

\$1. Introductory. The formula

$$
Q_{n}(z)=\frac{1}{2} \int_{-1}^{1} \frac{P_{n}(\mu)}{z-\mu} d \mu
$$

where $n$ is zero or a positive integer and $|z|>1$, was given by F. E. Neumann [Crelle's Journal, XXXVII (1848), p. 24]. In $\S 2$ of this paper some related formulae are given; the extension to the case when $n$ is not integral is dealt with in $\S 3$; while in $\S 4$ the corresponding formulae for the Associated Legendre Functions when the sum of the degree and the order is a positive integer are established.

$\S 2$. Related Formulae. It will be assumed throughout that $|z|>1$. From (1), if $m$ is a positive integer,

$$
\begin{aligned}
z^{m} Q_{n}(z)-\frac{1}{2} & \int_{-1}^{1} \frac{\mu^{m} P_{n}(\mu)}{z-\mu} d \mu \\
& =\frac{1}{2} \int_{-1}^{1} \frac{z^{m}-\mu^{m}}{z-\mu} P_{n}(\mu) d \mu \\
& =\frac{1}{2} \int_{-1}^{1}\left(z^{m-1}+z^{m-2} \mu+\ldots+\mu^{m-1}\right) P_{n}(\mu) d \mu .
\end{aligned}
$$

If $m \leqq n$, this last integral vanishes. Therefore

It follows that

$$
z^{m} Q_{n}(z)=\frac{1}{2} \int_{-1}^{1} \frac{\mu^{m} P_{n}(\mu)}{z-\mu} d \mu, m \leqq n
$$

$$
P_{m}(z) Q_{n}(z)=\frac{1}{2} \int_{-1}^{1} \frac{P_{m}(\mu) P_{n}(\mu)}{z-\mu} d \mu, m \leqq n .
$$

If $m=n+1$, (A) is equal to $\quad \frac{1}{2} \int_{-1}^{1} \mu^{n} P_{n}(\mu) d \mu$,

and this is equal to

$$
\frac{2^{n}(n !)^{2}}{(2 n) !} \frac{1}{2} \int_{-1}^{1}\left\{P_{n}(\mu)\right\}^{2} d \mu
$$

Thus

$$
z^{n+1} Q_{n}(z)=\frac{1}{2} \int_{-1}^{1} \frac{\mu^{n+1} P_{n}(\mu)}{z-\mu} d \mu+\frac{2^{n}(n !)^{2}}{(2 n+1) !}
$$

From this and (2) it follows that

$$
P_{n+1}(z) Q_{n}(z)=\frac{1}{2} \int_{-1}^{1} \frac{P_{n+1}(\mu) P_{n}(\mu)}{z-\mu} d \mu+\frac{1}{n+1} .
$$

This at once gives the known result

$$
P_{n+1}(z) Q_{n}(z)-P_{n}(z) Q_{n+1}(z)=\frac{1}{n+1}
$$

Other formulae of similar type are

$$
z P_{m}(z) Q_{n}(z)=\frac{1}{2} \int_{-1}^{1} \frac{\mu P_{m}(\mu) P_{n}(\mu)}{z-\mu} d \mu, m<n,
$$




$$
\begin{aligned}
z P_{n}(z) Q_{n}(z) & =\frac{1}{2} \int_{-1}^{1} \frac{\mu\left\{P_{n}(\mu)\right\}^{2}}{z-\mu} d \mu+\frac{1}{2 n+1}, \ldots \ldots . \\
\left(z^{2}-1\right) Q_{n}^{\prime}(z) & =\frac{1}{2} \int_{-1}^{1} \frac{\left(\mu^{2}-1\right) P_{n}^{\prime}(\mu)}{z-\mu} d \mu, n \geqq 1, \ldots \ldots . \\
z^{m}\left(z^{2}-1\right) Q_{n}^{\prime}(z) & =\frac{1}{2} \int_{-1}^{1} \frac{\mu^{m}\left(\mu^{2}-1\right) P_{n}^{\prime}(\mu)}{z-\mu} d \mu, m<n, \ldots . \\
\left(z^{2}-1\right) P_{m}(z) Q_{n}^{\prime}(z) & =\frac{1}{2} \int_{-1}^{1} \frac{\left(\mu^{2}-1\right) P_{m}(\mu) P_{n}^{\prime}(\mu)}{z-\mu} d \mu, m<n .
\end{aligned}
$$

§ 3. Extension to General Values of $n$. If $n$ is not integral and $-1<\mu<1$,

Thus, if $|z|>1$,

$$
P_{n}(\mu)=\frac{\sin n \pi}{\pi} \sum_{p=0}^{\infty}(-1)^{p}\left(\frac{1}{n-p}-\frac{1}{n+p+1}\right) P_{p}(\mu)
$$

$$
\frac{1}{2} \int_{-1}^{1} \frac{P_{n}(\mu)}{z-\mu} d \mu=\frac{\sin n \pi}{\pi} \sum_{p=0}^{\infty}(-1)^{p}\left(\frac{1}{n-p}-\frac{1}{n+p+1}\right) Q_{p}(z),
$$

the series on the right being absolutely convergent $*$ for $|z|>1$. When $n \rightarrow p$ this formula reduces to (1) with $p$ in place of $n$.

The term by term integration requires justification, as the series on the right of (12) is divergent when $\mu=-1$. The proof is based on the formula

$$
\begin{aligned}
& P_{p}(\cos \theta)=\frac{1}{\sqrt{ }(2 \pi \sin \theta)} \frac{\Gamma(p+1)}{\Gamma\left(p+\frac{3}{2}\right)} \\
& \times \sum_{i,-i} e^{\frac{1}{2 \pi i-\left(p+\frac{1}{2}\right) \theta i}} F\left(\frac{1}{2}, \frac{1}{2} ; p+\frac{3}{2} ;-\frac{e^{-i \theta}}{2 i \sin \theta}\right) .
\end{aligned}
$$

Here the hypergeometric series converges for $\frac{1}{6} \pi \leqq \theta \leqq \frac{5}{6} \pi$ and is asymptotic in $p$ for the other values of $\theta$ in the range $0<\theta<\pi$. The series on the right of (12) therefore converges absolutely and uniformly for $0<\epsilon \leqq \theta \leqq \pi-\epsilon$.

Again, if $|\zeta|<1$,

$$
F\left(\frac{1}{2}, \frac{1}{2} ; p+\frac{3}{2} ; \zeta\right)=\frac{\Gamma\left(p+\frac{3}{2}\right)}{\Gamma\left(\frac{1}{2}\right) \Gamma(p+1)} \int_{0}^{1} t^{-\frac{1}{2}}(1-t)^{p}(1-\zeta t)^{-\frac{1}{2}} d t,
$$

and the expression on the right may be used to obtain the analytical continuation of the left-hand side over the $\zeta$-plane bounded by a cross-cut along the real axis from 1 to $+\infty$.

Now, if

$$
\begin{aligned}
\zeta & =-e^{-i \theta} /(2 i \sin \theta)=\frac{1}{2}-\frac{1}{2 i} \cot \theta, \\
|1-\zeta t| & =\left|1-\frac{1}{2} t-\frac{1}{2} i t \cot \theta\right| \\
& =\sqrt{ }\left(1-t+\frac{1}{4} t^{2} \operatorname{cosec} 2 \theta\right) \\
& =\sqrt{ }\left\{\cos { }^{2} \theta+\left(\sin \theta-\frac{1}{2} t \operatorname{cosec} \theta\right)^{2}\right\} \\
& \geqq|\cos \theta| .
\end{aligned}
$$

From (15) it follows that, if $0<\theta \leqq \epsilon$ or $\pi-\epsilon \leqq \theta<\pi$,

Therefore, from (14),

$$
\left|F\left(\frac{1}{2}, \frac{1}{2} ; p+\frac{3}{2} ;-\frac{e^{-i \theta}}{2 i \sin \theta}\right)\right| \leqq \sqrt{ }(\sec \theta) .
$$

$$
\left|\sin \theta P_{p}(\cos \theta)\right| \leqq \sqrt{ }\left(\frac{\sin \theta}{2 \pi}\right) \frac{\Gamma(p+1)}{\Gamma\left(p+\frac{3}{2}\right)} 2 \sqrt{ }(\sec \epsilon),
$$

and, by continuity, this holds for $0 \leqq \theta \leqq \epsilon$ and for $\pi-\epsilon \leqq \theta \leqq \pi$.

* Cf. Phil. Mag., Ser. 7, XXXV (1944), p. 673-6. 
It follows that the series on the right of (12), multiplied by $\sin \theta$, where $\mu=\cos \theta$, converges absolutely and uniformly for $0 \leqq \theta \leqq \pi$ and that the term by term integration is justified.

§ 4. Legendre Functions when the sum of the Degree and the Order is a Positive Integer. If $n$ is zero or a positive interger,

and

$$
\begin{aligned}
P_{m+n}^{-m}(z)= & \frac{2^{m+n} \Gamma\left(m+n+\frac{1}{2}\right)}{\Gamma\left(\frac{1}{2}\right) \Gamma(2 m+n+1)}\left(z^{2}-1\right)^{\ddagger m} \\
& \times\left\{z^{n}-\frac{n(n-1)}{2(2 m+2 n-1)} z^{n-2}+\ldots\right\}
\end{aligned}
$$

$\quad T_{m+n}^{-m}(\mu)=\frac{(-1)^{n}\left(1-\mu^{2}\right)^{-1} m}{2^{m+n} \Gamma(m+n+1)} \frac{d^{n}}{d \mu^{n}}\left\{\left(1-\mu^{2}\right)^{m+n}\right\}$

the latter formula being an extension of Rodrigues' Formula.

By using (17) and integrating by parts it can be shown that, if $p$ is a positive integer,

The formula*

$$
\begin{aligned}
\int_{-1}^{1} \mu^{p}\left(1-\mu^{2}\right)^{\frac{1}{2} m} & T_{m+n}^{-m}(\mu) d \mu \\
& =\left\{\begin{array}{ll}
0, \quad p<n, \\
\frac{\Gamma\left(\frac{1}{2}\right) \cdot n !}{2^{m+n} \Gamma\left(m+n+\frac{3}{2}\right)}
\end{array}, p=n .\right.
\end{aligned}
$$

$$
\left(z^{2}-1\right)^{\frac{1}{m} m} Q_{m+n}^{-m}(z)=\frac{1}{2} \int_{-1}^{1} \frac{\left(1-\mu^{2}\right)^{\frac{1}{2} m} T_{m+n}^{-m}(\mu)}{z-\mu} d \mu
$$

is proved by expanding on the right in descending powers of $z$ and using (18). It then follows that, if $p$ is a positive integer,

$$
\begin{aligned}
& z^{p}\left(z^{2}-1\right)^{\frac{1}{m}} Q_{m+n}^{-m}(z)=\frac{1}{2} \int_{-1}^{1} \frac{\mu^{p}\left(1-\mu^{2}\right)^{\frac{1}{2} m} T_{m+n}^{-m}(\mu)}{z-\mu} d \mu, \quad p \leqq n, \\
& P_{m+p}^{-m}(z) Q_{m+n}^{-m}(z)=\frac{1}{2} \int_{-1}^{1} \frac{T_{m+p}^{-m}(\mu) T_{m+n}^{-m}(\mu)}{z-\mu} d \mu, \quad p \leqq n, \\
& z^{n+1}\left(z^{2}-1\right)^{\frac{1}{m} m} Q_{m+n}^{-m}(z)=\frac{1}{2} \int_{-1}^{1} \frac{\mu^{n+1}\left(1-\mu^{2}\right)^{\frac{1}{2} m} T_{m+n}^{-m}(\mu)}{z-\mu} d \mu \\
& +\frac{\Gamma\left(\frac{1}{2}\right) \cdot n !}{2^{m+n+1} \Gamma\left(m+n+\frac{3}{2}\right)}, \\
& P_{m+n+1}^{-m}(z) Q_{m+n}^{-m}(z)=\frac{1}{2} \int_{-1}^{1} \frac{T_{m+n+1}^{-m}(\mu) T_{m+n}^{-m}(\mu)}{z-\mu} d \mu \\
& +\frac{n !}{\Gamma(2 m+n+2)} \text {, } \\
& P_{m+n+1}^{-m}(z) Q_{m+n}^{-m}(z)-P_{m+n}^{-m}(z) Q_{m+n+1}^{-m}(z)=\frac{n !}{\Gamma(2 m+n+2)}
\end{aligned}
$$

UNIVERSTTY OF GLASGOW 